\title{
Nonlinear and Complex Dynamics in Real Systems
}

\author{
William A. Barnett
}

Department of Economics, University of Kansas, Lawrence, Kansas.

\section{Apostolos Serletis}

Department of Economics, University of Calgary, Calgary, Alberta.

\section{Demitre Serletis}

Division of Neurosurgery, University of Toronto, Toronto, Ontario.

\begin{abstract}
In this article we provide a review of the literature with respect to fluctuations in real systems and chaos. In doing so, we contrast the order and organization hypothesis of real systems to nonlinear chaotic dynamics and discuss some techniques used in distinguishing between stochastic and deterministic behavior. Moreover, we look at the issue of where and when the ideas of chaos could profitably be applied to real systems.
\end{abstract}

Keywords: Chaos; Nonlinearity; Self-organized criticality.

\section{Introduction}

As Århem, Blomberg, and Liljenström (2000, p. ix) put it in their Preface to their edited volume, Disorder Versus Order in Brain Function,

"life is associated with a high degree of order and organization. However, disorder in various contexts referred to as fluctuations, noise or chaos is also a crucial component of many biological processes. In evolution, random errors in the reproduction of the genetic material provides a variation that is fundamental for the selection of adaptive organisms. At a molecular level, thermal fluctuations govern the movements and functions of the macromolecules in the cell. Yet, such problems have been remarkably little studied and a critical analysis of the positive and negative effects of disorder for the living systems is much needed."

Our purpose in this paper is to provide a review of recent state-of-the-art developments in dynamical systems theory and their relevance and usefulness in studying real systems in a variety of disciplines, such as astronomy, biology, and economics. In doing so, we shall concentrate on the ideas of chaos --- i.e., deterministic systems with stochastic behavior. It is now well known that perfectly deterministic systems (i.e., systems with no stochastic components) of low dimensions (i.e., with a small number of state variables) and with simple nonlinearities (i.e., a single quadratic function) can have stochastic behavior. The discovery that such systems exist has brought about a profound reconsideration of the issue of randomness.

Moreover, as Schiff et al. (1994, p. 615) argue,

"following the recent theoretical prediction that chaotic physical systems might be controllable with small 
perturbations, there has been rapid and successful application of this technique to mechanical systems, electrical circuits, lasers and chemical reactions. Following the demonstration of the control of chaos in arrhythmic cardiac tissue, there are no longer any technical barriers to applying these techniques to neural tissue."

In the following sections we shall try to make the basic notions of chaos and other related concepts more precise, having in mind their actual or potential applications to real systems. In doing so, we shall take the geometric (or topological) approach to the study of dynamical systems, based on the theory of differential/difference equations. This approach, has been very successful in the study of low-dimensional systems (systems with one and perhaps two variables). For higher-dimensional systems, however, a different approach based on the axiomatic formulation of probability theory and aimed at the investigation of the statistical properties of chaos, known as the ergodic approach, is more suitable. A discussion of dynamical systems from an ergodic point of view is well beyond the scope of this article.

\section{Dynamical Systems}

Dynamical systems, occurring in biology, meteorology, climate, and possibly economics and finance, can be modelled with deterministic time evolutions that have either a continuous time

$$
\dot{x}=f(x), \quad x \in \mathrm{R}^{n}
$$

or discrete time

$$
x_{t+1}=T\left(x_{t}\right), \quad x \in \mathrm{R}^{n}, t \in \mathbb{N} .
$$

Equations like (2) are often referred to as iterated maps since their orbits are obtained recursively given an initial condition $x_{\mathrm{t}}$. For example, if we compose $T$ with itself, then we get the second iterate

$$
x_{t+2}=T \circ T\left(x_{t}\right)=T^{2}\left(x_{t}\right)
$$

and by induction on $n$ we get the $n$th iterate

$$
x_{t+n}=T \circ T^{n-1}\left(x_{t}\right)=T^{n}\left(x_{t}\right) .
$$

Hence, by the notation $T^{n}(x)$, we mean $T$ composed with itself $n-1$ times --- not the $n$th derivative of $T$ or the $n$th power of $T$.

Notice the following difference between the orbits of continuous-time and those of discrete-time systems: the former are continuous curves in the state space, whereas the latter are sequences of points in space. Also, the fact that a map is a function implies that, starting from any given point in space, there exists only one forward orbit. If the function is non-invertible, however, backward orbits are not defined. In this essay we focus on deterministic time evolutions that have a discrete time.

\section{Strange Attractors ('Fractal Sets')}

To discuss recurrence properties of orbits of a dynamical system, we shall start from the notion of attractors. The simplest type of an attractor is a stable fixed point also known as a stable equilibrium. Ascertaining the existence of a fixed/equilibrium point mathematically amounts to finding the solutions of a system of algebraic equations. Let's consider the one-dimensional 'logistic map,' one of the most common type of dynamical system encountered in applications of chaos theory

$$
x_{t+1}=r x_{t}\left(1-x_{t}\right)
$$

with $x \in[0,1]$ and $r \in(0,4]$. To find the fixed points of (3), we put $x_{t+1}=x_{t}=\bar{x}$ and solve for $\bar{x}$, finding $\bar{x}_{1}=0$ and $\bar{x}_{2}=1-1 / r$.

In general, we can examine the dynamical information contained in the derivative of the map at the fixed point, $T^{\prime}(\bar{x})$. If $\left|T^{\prime}(\bar{x})\right| \neq 1$, $\bar{X}$ is called hyperbolic fixed point. In fact a fixed point $\bar{X}$ is stable (or attracting) if $\left|T^{\prime}(\bar{x})\right|<1$, 
unstable (or repelling) if $\left|T^{\prime}(\bar{x})\right|>1$, and superstable (or superattractive) if $\left|T^{\prime}(\bar{x})\right|=1$ --- superstable in the sense that convergence to the fixed point is very rapid. Fixed points whose derivatives are equal to one in absolute value are called nonhyperbolic (or neutral) fixed points.

Next in the scale of complexity of invariant sets, we consider stable periodic solutions, or limit cycles. For maps, a point $\bar{x}$ is a periodic point of $T$ with period $k$, if $T^{k}(\bar{x})=\bar{x}$ for $k>1$ and $T^{j}(\bar{x}) \neq \bar{x}$ for $0<j<k$. In other words, $\bar{x}$ is a periodic point of $T$ with period $k$ if it is a fixed point of $T^{k}$. In this case we say that $\bar{X}$ has period $k$ under $T$, and the orbit is a sequence of $k$ distinct points $\left\{\bar{X}, T(\bar{x}), \ldots, T^{k}(\bar{x})\right\}$ which, under the iterated action of $T$, are repeatedly visited by the system, always in the same order. Since all points between $\bar{x}$ and $T^{k}(\bar{x})$ are also period $k$ points, the resulting sequence is known as a period $k$ cycle or alternatively a k-period cycle. Notice that $k$ is the least period --- if $k=1$, then $\bar{x}$ is a fixed point for $T$.

The third basic type of attractor is called quasiperiodic. If we consider the motion of a dynamical system after all transients have died out, the simplest way of looking at a quasiperiodic attractor is to describe its dynamics as a mechanism consisting of two or more independent periodic motions --- see Hilborn (1994, pp. 154-157) for a non-technical discussion. Quasiperiodic orbits can look quite complicated, since the motion never exactly repeats itself (hence, quasi), but the motion is not chaotic (as it was wrongly once conjectured).

Attractors with an orbit structure more complicated than that of periodic or quasiperiodic systems are called chaotic or strange attractors. The strangeness of an attractor mostly refers to its geometric characteristic of being a 'fractal' set, whereas chaotic often refers to a dynamic property, known as 'sensitive dependence on initial conditions,' or equivalently, 'divergence of nearby orbits.' Notice that strangeness, as defined by fractal dimension, and chaoticity, as defined by sensitive dependence on initial conditions, are independent properties. Thus, we have chaotic attractors that are not fractal and strange attractors that are not chaotic.

As we shall see, separation of nearby orbits, or, equivalently, amplification of errors is the basic mechanism that makes accurate prediction of the future course of chaotic orbits impossible, except in the short run. On the other hand, as chaotic attractors are bounded objects, the expansion that characterizes their orbits must be accompanied by a 'folding' action that prevents them from escaping to infinity. The coupling of 'stretching and folding' of orbits is the distinguishing feature of chaos, and it is at the root of both the complexity of its dynamics and the 'strangeness' of its geometry.

In what follows, we shall briefly discuss the 'fractal' property of chaotic attractors, whereas the 'sensitive dependence on initial conditions' property of chaos will be given greater attention in the next section; this property of chaos is, in our opinion, the most relevant in real systems.

\section{Fractal Dimension}

The term 'fractal,' coined by Mandelbrot (1985), refers to geometrical objects characterized by non-integral dimensions and 'self-similarity.' Intuitively, a snowflake can be taken as a natural fractal. The problem of defining measurement criteria finer than the familiar Euclidean dimensions (length, area, volume) in order to quantify the geometric properties of 'broken' or 'porous' objects was tackled by mathematicians long before the name and properties of fractals became popular. There now exists a rather large number of criteria for measuring qualities that otherwise have no clear definition (such as, for example, the degree of roughness or brokenness of an object), but we shall limit ourselves here to discuss the simplest type concisely.

Let $S$ be a set of points in a space of Euclidean dimension $p$ (think, for example, of the points on the real line generated by the iterations of a one-dimensional map). We now consider certain boxes of side $\varepsilon$ (or, equivalently, certain spheres of radius $\varepsilon$ ), and 
calculate the minimum number of such cells, $N(\varepsilon)$, necessary to 'cover' $S$. Then, the fractal dimension $D$ of the set $S$ will be given by the following limit (assuming it exists)

$$
D=\lim _{\varepsilon \rightarrow 0} \frac{\log (N(\varepsilon))}{\log (1 / \varepsilon)}
$$

The quantity defined in equation (4) is also called the (Kolmogorov) capacity dimension. It is easily seen that, for the most familiar geometrical objects, it provides perfectly intuitive results. For example, if $S$ consists of just one point, $N(\varepsilon)=1$ and $D=0$; if it is a segment of unit length, $N(\varepsilon)=1 / \varepsilon$, and $D=1$; if it is a plane of unit area, $N(\varepsilon)=1 / \varepsilon^{2}$ and $D=2$; finally, if $S$ is a cube of unit area, $N(\varepsilon)=1 / \varepsilon^{3}$ and $D=3$, etc. That is to say, for 'regular' geometric objects, dimension $D$ does not differ from the usual Euclidean dimension, and in particular, $D$ is an integer.

The concept of fractal dimension is useful in the geometric analysis of dynamical systems, because it can be conceived of as a measure of the way trajectories fill the phase space under the action of a flow or a map. A non-integer fractal dimension, for example, indicates that trajectories of a system fill up less than an integer subspace of the phase space. Also, the concept of fractal dimension is useful in the quantitative analysis of chaotic attractors. For example, the dimension of the attractor of a system, as measured by (4), can be taken as an index of complexity, as indicated by the essential dimension of the system.

\section{Lyapunov Exponents}

As already noted, sensitive dependence on initial conditions is the most relevant property of chaos and its characterization in terms of Lyapunov exponents is the most satisfactory from a computable perspective. Lyapunov exponents measure average exponential divergence or convergence between trajectories that differ only in having an infinitesimally small' difference in their initial conditions and remain well-defined for noisy systems. A bounded system with a positive global Lyapunov exponent is one operational definition of chaotic behavior and reflects the impossibility of long-term predictability.

Although Lyapunov exponents could be discussed in a rather general framework, we shall deal with the issue in the context of one-dimensional maps, since they are by far the most common type of dynamical system encountered in applications of chaos theory. Consider, therefore, the discrete time map

$$
x_{t+1}=T\left(x_{t}\right), \quad x \in \mathrm{R}^{n}, t \in \mathbb{N} \text {. }
$$

We want to describe the evolution in time of two orbits originating from two nearby points $x_{0}$ and $x_{0}+\varepsilon$ (where $\varepsilon$ is the difference, assumed to be infinitesimally small, between $x_{0}$ and $\left.x_{0}+\varepsilon\right)$. If we apply the map function $T, n$ times to each point, the difference between the results will be related to $x_{0}$ as follows

$$
d_{n}=e^{n \lambda\left(x_{0}\right)} \varepsilon,
$$

where $d_{n}$ is the difference between the two points after they have been iterated by the map $T$, $n$ times and $\lambda\left(x_{0}\right)$ is the rate of convergence or divergence.

Taking the logarithm of the above equation and solving for $\lambda\left(x_{0}\right)$, asymptotically we shall have

$$
\begin{aligned}
\lambda\left(x_{0}\right) & =\lim _{n \rightarrow \infty} \frac{1}{n} \log \left|\frac{d_{n}}{\varepsilon}\right| \\
& =\lim _{n \rightarrow \infty} \frac{1}{n} \log \left|T^{\prime}\left(x_{n-1}\right) \ldots T^{\prime}\left(x_{1}\right) T^{\prime}\left(x_{0}\right)\right| \\
& =\lim _{n \rightarrow \infty} \frac{1}{n} \sum_{j=0}^{n-1} \log \left|T^{\prime}\left(x_{j}\right)\right| .
\end{aligned}
$$

The quantity $\lambda\left(x_{0}\right)$ is called Lyapunov exponent. Note that the right hand side of (6) is an average along an orbit (a time average) of the logarithm of the derivative. From equation (6), 
the interpretation of $\lambda\left(x_{0}\right)$ is straightforward: it is the (local) asymptotic exponential rate of divergence of nearby orbits.

The sign of Lyapunov exponents is especially important to classify different types of dynamical behavior. In particular, the presence of a positive Lyapunov exponent signals that nearby orbits diverge exponentially in the corresponding direction. In fact, the presence of a positive Lyapunov exponent is intimately related to the lack of predictability of dynamical systems, and thus it is an essential feature of chaotic behavior.

\section{Where Can Chaos be Applied?}

In recent years, following the theoretical prediction that chaotic systems might be controllable by changing their bifurcation parameters, there has been a number of interesting applications of chaos theory to biological systems --- see, for example, Garfinkel et al. (1992) for an application to cardiac chaos and Schiff et al. (1994) regarding chaos in the brain. The application, however, of chaos theory to finance, economics, and social science phenomena has not, so far, produced especially useful results, although successful tests for the existence of chaos have been reported, such as Barnett and Chen (1988) and Serletis and Gogas (1997). The evidence so far is against low-dimensional chaos in economic and financial time series (stock prices, interest rates, and exchange rates). Of course, the failure to detect low-dimensional chaos does not preclude the possibility of high-dimensional chaos in these variables. The presence, however, of dynamic noise makes it difficult and perhaps impossible to distinguish between (noisy) high-dimensional chaos and pure randomness.

In fact, meaningful analyses of real systems (heart, brain, economy, planetary orbits) in terms of chaos theory should consider a number of theoretical and practical issues. As Ruelle (1994, p. 27) puts it

"real systems can in general be described as deterministic systems with some added noise. This description is sufficiently vague that it appears to cover everything. In economics, for instance, such a description is familiar and the noise is called 'shocks.' A first remark concerning the above picture is that the separation between noise and the deterministic part of the evolution is ambiguous, because one can always interpret 'noise' as a deterministic time evolution in infinite dimension."

Hence, the possible existence of chaos could be exploitable and even invaluable if the deterministic part of the system is low-dimensional and its noisy part is of a small amplitude. Moreover, it is useful to know how the noise comes into the deterministic signal. For example, an additive noise contribution could be easily removed from a low-dimensional deterministic signal, even if its amplitude is large, thereby rendering short-run, nonlinear-based prediction and control possible. Of course, prediction and control over long periods is impossible, since they are ruined by the sensitive dependence on initial conditions property of chaos.

Although low-dimensionality is crucial in the reconstruction of the dynamics of a deterministic dynamical system, knowledge of the fundamental (difference or differential) equations of time evolution is necessary for effective short-run prediction and control. In the case of astronomy, for example, such equations are known with great precision, providing reliable models with a prediction time on the order of thousands of years. However, dynamical systems occurring in meteorology, climate, biology, and possibly economics, finance, and social science phenomena are more perplexing, with interesting time evolutions, but much shorter prediction times. In meteorology, for example, chaotic models have a prediction time on the order of a few days, and models in climate seem to have the same problem for the same reasons as well as the added problem of time-varying parameters.

Overall, regarding the relevance and usefulness of dynamical systems theory, as Ruelle (1994, p. 30) puts it, 
"the theory of dynamical systems has made important contributions to our understanding of the 'real world,' and in particular the role played in it by 'chaos.' One can expect further valuable contributions, especially in the difficult domain of biology. But a necessary condition for progress is that the relations between models and the real world be properly assessed."

\section{Conclusion}

We have provided a brief review of the recent literature about the ideas of chaos. Chaos is a nonlinear deterministic process which looks random. In fact, chaotic processes have first and second moment properties that are the same as for white noise processes. The distinguishing feature of chaotic systems, however, is that they exhibit sensitive dependence on initial conditions, meaning that nearby identical chaotic systems in slightly different states will rapidly evolve toward very different states. We have also argued that chaos is important since evidence of chaos implies that (nonlinearity-based) prediction is possible, at least in the short run and provided that the actual generating mechanism is known exactly. In the long run, chaos implies that prediction is all but impossible due to sensitive dependence on initial conditions.

But as already noted, the relations between our models and the real world should be properly assessed for further significant contributions regarding the workings of real systems. In this regard we should also note that there is a second type of nonlinear process, known as self-organized criticality, recently discovered in physics by Bak et al. (1987). Self-organized criticality applies to large interactive systems such as the human body and the stock market. Unlike chaos, however, self-organized criticality is a probabilistic process. It incorporates a dominant long-run trend toward greater sensitivity and a short-run catastrophic element, which is triggered by random shocks within the system. There is thus another issue, that of whether real systems are chaotic or self-organized critical.

\section{References}

[1] Árhem, Peter, Clas Blomberg, and Hans Liljenström. Disorder Versus Order in Brain Function. World Scientific (2000).

[2] Bak, P., C. Tang, and K. Wiesenfeld. "Self-Organized Criticality: An Explanation of 1/f Noise." Physical Review Letters 59 (1987), 381-384.

[3] Barnett, Willian A. and Ping Chen. "The Aggregation-Theoretic Monetary Aggregates are Chaotic and Have Strange Attractors: An Econometric Application of Mathematical Chaos." In Barnett, W.A., E. Berndt, and H. White (eds.), Dynamic Econometic Modelling. Cambridge: Cambridge University Press (1988).

[4] Garfinkel, Alan, Mark L. Spano, William L. Ditto, and JamesN. Weiss. "Controlling Cardiac Chaos.” Science 257 (1992), 1230-1235.

[5] Hilborn, Robert C. Chaos and Nonlinear Dynamics: An Introduction for Scientists and Engineers. New York: Oxford University Press (1994).

[6] Mandelbrot, Benoit B. "Self-Affine Fractals and Fractal Dimension." Physica Scripta 32 (1985), 257-260.

[7] Ruelle, David. "Where Can One Hope to Profitably Apply the Ideas of Chaos?" Physics Today 47 (1994), 24-30.

[8] Schiff, Steven J., Kristin Jerger, Duc H. Duong, Taeun Chang, Mark L. Spano, and William L. Ditto. "Controlling Chaos in the Brain." Nature 370 (1994), 615-620.

[9] Serletis, Apostolos and Periklis Gogas. "Chaos in East European Black Market Exchange Rates." Research in Economics 51 (1997), 359-385. 